\title{
Erratum to: Cementless total hip arthroplasty in the treatment after acetabular fractures
}

\author{
Wael Salama $^{1,2} \cdot$ Paolo Ditto $^{2} \cdot$ Shazly Mousa $^{1} \cdot$ Abdelrahman Khalefa $^{1} \cdot$ \\ Ahmed Sleem $^{1} \cdot$ Laura Ravera $^{2} \cdot$ Alessandro Masse $^{2}$
}

Published online: 6 September 2017

(c) Springer-Verlag France SAS 2017

\section{Erratum to: Eur J Orthop Surg Traumatol DOI 10.1007/s00590-017-2021-x}

The author would like to correct the errors in the publication of the original article. The corrected details are given below for your reading.

In "Patients and methods" section, third paragraph should read as:

The initial acetabular fracture pattern was classified according to the system of Judet et al. [8]; there were nine cases of simple type and twelve cases of associated type. Seventeen patients were treated by ORIF, while 4 were managed conservatively.

In "Surgical technique" section, first paragraph should read as:
All patients were surgically exposed by using Kocher-Langenbeck approach, and the hardware was partially removed in three patients and left in situ in 18 patients. Swap for culture and sensitivity was done when infection was suspected.

In "Discussion" section, last sentence of the second paragraph should read as:

In fact, their data demonstrated cup revision rate of $13.7 \%$ and loosening rate of $49 \%$ with $27 \%$ of those hips having symptomatic loosening.

The online version of the original article can be found under doi:10.1007/s00590-017-2021-x.

Wael Salama

Waeladel582@gmail.com

1 Orthopedic Department, Faculty of Medicine, Sohag University Hospital (SUH), Sohag 82524, Egypt

2 Pelvic Unit, San Luigi Hospital of Orbassano, Faculty of Medicine and Surgery, University of Turin, 10043 Turin, Italy 